\title{
First Measurement of Transverse-Spin-Dependent Azimuthal Asymmetries in the Drell-Yan Process
}

M. Aghasyan,${ }^{24}$ R. Akhunzyanov, ${ }^{7}$ G. D. Alexeev, ${ }^{7}$ M. G. Alexeev, ${ }^{25}$ A. Amoroso, ${ }^{25,26}$ V. Andrieux,${ }^{27,20}$ N. V. Anfimov, ${ }^{7}$ V. Anosov, ${ }^{7}$ A. Antoshkin, ${ }^{7}$ K. Augsten, ${ }^{78}$ W. Augustyniak, ${ }^{28}$ A. Austregesilo, ${ }^{15}$ C. D. R. Azevedo, ${ }^{1}$ B. Badełek, ${ }^{29}$ F. Balestra, ${ }^{25,26}$ M. Ball, ${ }^{3}$ J. Barth, ${ }^{4}$ R. Beck, ${ }^{3}$ Y. Bedfer, ${ }^{20}$ J. Bernhard, ${ }^{12,9}$ K. Bicker, ${ }^{15,9}$ E. R. Bielert, ${ }^{9}$ R. Birsa, ${ }^{24}$ M. Bodlak,${ }^{17}$ P. Bordalo, ${ }^{11, b}$ F. Bradamante, ${ }^{23,24}$ A. Bressan, ${ }^{23,24}$ M. Büchele, ${ }^{8}$ W.-C. Chang, ${ }^{21}$ C. Chatterjee, ${ }^{6}$ M. Chiosso, ${ }^{25,26}$ I. Choi, ${ }^{27}$ S.-U. Chung, ${ }^{15, \mathrm{c}}$ A. Cicuttin, ${ }^{24, \mathrm{~d}}$ M. L. Crespo, ${ }^{24, \mathrm{~d}}$ S. Dalla Torre, ${ }^{24}$ S. S. Dasgupta, ${ }^{6}$ S. Dasgupta, ${ }^{23,24}$ O. Yu. Denisov, ${ }^{26}$ L. Dhara, ${ }^{6}$ S. V. Donskov, ${ }^{19}$ N. Doshita,${ }^{31}$ Ch. Dreisbach, ${ }^{15}$ W. Dünnweber, ${ }^{e}$ M. Dziewiecki, ${ }^{30}$ A. Efremov, ${ }^{7}$ P. D. Eversheim, ${ }^{3}$ M. Faessler, ${ }^{\text {e }}$ A. Ferrero, ${ }^{20}$ M. Finger, ${ }^{17}$ M. Finger Jr, ${ }^{17}$ H. Fischer, ${ }^{8}$ C. Franco, ${ }^{11}$ N. du Fresne von Hohenesche, ${ }^{12,9}$ J. M. Friedrich, ${ }^{15}$ V. Frolov ${ }^{7,9}$ E. Fuchey, ${ }^{20}$ F. Gautheron, ${ }^{2}$ O. P. Gavrichtchouk, ${ }^{7}$ S. Gerassimov, ${ }^{14,15}$ J. Giarra, ${ }^{12}$ F. Giordano, ${ }^{27}$ I. Gnesi, ${ }^{25,26}$ M. Gorzellik, ${ }^{8}$ A. Grasso, ${ }^{25,26}$ M. Grosse Perdekamp ${ }^{27}$ B. Grube, ${ }^{15}$ T. Grussenmeyer, ${ }^{8}$ A. Guskov, ${ }^{7}$ D. Hahne, ${ }^{4}$ G. Hamar, ${ }^{24}$ D. von Harrach, ${ }^{12}$ F. H. Heinsius ${ }^{8}$ R. Heitz ${ }^{27}$ F. Herrmann,${ }^{8}$ N. Horikawa, ${ }^{16, f}$ N. d'Hose, ${ }^{20}$ C.-Y. Hsieh, ${ }^{21, g}$ S. Huber, ${ }^{15}$ S. Ishimoto,${ }^{31, h}$ A. Ivanov, ${ }^{25,26}$ Yu. Ivanshin, ${ }^{7}$ T. Iwata, ${ }^{31}$ V. Jary,${ }^{18}$ R. Joosten, ${ }^{3}$ P. Jörg, ${ }^{8}$ E. Kabuß, ${ }^{12}$ A. Kerbizi, ${ }^{23,24}$ B. Ketzer, ${ }^{3}$ G. V. Khaustov, ${ }^{19}$ Yu. A. Khokhlov, ${ }^{19, i}$ Yu. Kisselev, ${ }^{7}$ F. Klein, ${ }^{4}$ J. H. Koivuniemi, ${ }^{2,27}$ V. N. Kolosov, ${ }^{19}$ K. Kondo, ${ }^{31}$ K. Königsmann, ${ }^{8}$ I. Konorov, ${ }^{14,15}$ V. F. Konstantinov, ${ }^{19}$ A. M. Kotzinian,,${ }^{25,26}$ O. M. Kouznetsov, ${ }^{7}$ Z. Kral, ${ }^{18}$ M. Krämer, ${ }^{15}$ P. Kremser, ${ }^{8}$ F. Krinner, ${ }^{15}$ Z. V. Kroumchtein, ${ }^{7, a}$ Y. Kulinich, ${ }^{27}$ F. Kunne,${ }^{20}$ K. Kurek, ${ }^{28}$ R. P. Kurjata, ${ }^{30}$ A. Kveton, ${ }^{18}$ A. A. Lednev, ${ }^{19, a}$ M. Levillain, ${ }^{20}$ S. Levorato, ${ }^{24}$ Y.-S. Lian, ${ }^{21, j}$ J. Lichtenstadt, ${ }^{22}$ R. Longo, ${ }^{25,26}$ A. Maggiora, ${ }^{26}$ A. Magnon, ${ }^{27}$ N. Makins, ${ }^{27}$ N. Makke, ${ }^{24, d}$ G. K. Mallot, ${ }^{9}$ B. Marianski, ${ }^{28}$ A. Martin, ${ }^{23,24}$ J. Marzec, ${ }^{30}$ J. Matoušek, ${ }^{23,24,17}$ H. Matsuda, ${ }^{31}$ T. Matsuda, ${ }^{13}$ G. V. Meshcheryakov, ${ }^{7}$ M. Meyer, ${ }^{27,20}$ W. Meyer, ${ }^{2}$ Yu. V. Mikhailov, ${ }^{19}$ M. Mikhasenko, ${ }^{3}$ E. Mitrofanov, ${ }^{7}$ N. Mitrofanov, ${ }^{7}$ Y. Miyachi, ${ }^{31}$ A. Nagaytsev, ${ }^{7}$ F. Nerling, ${ }^{12}$ D. Neyret, ${ }^{20}$ J. Nový, ${ }^{18,9}$ W.-D. Nowak, ${ }^{12}$ G. Nukazuka, ${ }^{31}$ A. S. Nunes ${ }_{11}^{11}$ A. G. Olshevsky, ${ }^{7}$ I. Orlov ${ }^{7}$ M. Ostrick, ${ }^{12}$ D. Panzieri, ${ }^{26, k}$ B. Parsamyan, ${ }^{25,26}$ S. Paul, ${ }^{15}$ J.-C. Peng, ${ }^{27}$ F. Pereira, ${ }^{1}$ M. Pešek, ${ }^{17}$ D. V. Peshekhonov, ${ }^{7}$ N. Pierre ${ }^{12,20}$ S. Platchkov, ${ }^{20}$ J. Pochodzalla, ${ }^{12}$ V. A. Polyakov, ${ }^{19}$ J. Pretz,${ }^{4,1}$ M. Quaresma, ${ }^{11}$ C. Quintans, ${ }^{11}$ S. Ramos, ${ }^{11, b}$ C. Regali, ${ }^{8}$ G. Reicherz, ${ }^{2}$ C. Riedl, ${ }^{27}$ N. S. Rogacheva, ${ }^{7}$ M. Roskot,${ }^{17}$ D. I. Ryabchikov, ${ }^{19,15}$ A. Rybnikov, ${ }^{7}$ A. Rychter, ${ }^{30}$ R. Salac, ${ }^{18}$ V. D. Samoylenko, ${ }^{19}$ A. Sandacz, ${ }^{28}$ C. Santos, ${ }^{24}$ S. Sarkar ${ }^{6}$ I. A. Savin, ${ }^{7}$ T. Sawada, ${ }^{21}$ G. Sbrizzai,${ }^{23,24}$ P. Schiavon, ${ }^{23,24}$ K. Schmidt ${ }^{8}$ H. Schmieden, ${ }^{4}$ K. Schönning, ${ }^{9, m}$ E. Seder, ${ }^{20}$ A. Selyunin, ${ }^{7}$ O. Yu. Shevchenko, ${ }^{7, a}$ L. Silva, ${ }^{11}$ L. Sinha, ${ }^{6}$ S. Sirtl, ${ }^{8}$ M. Slunecka, ${ }^{7}$ J. Smolik, ${ }^{7}$ A. Srnka, ${ }^{5}$ D. Steffen,, 15 M. Stolarski, ${ }^{11}$ O. Subrt, ${ }^{9,18}$ M. Sulc, ${ }^{10}$ H. Suzuki, ${ }^{31, f}$ A. Szabelski, ${ }^{23,24,28}$ T. Szameitat ${ }^{8}$ P. Sznajder, ${ }^{28}$ S. Takewaka, ${ }^{25,26}$ M. Tasevsky, ${ }^{7}$ S. Tessaro, ${ }^{24}$ G. Terça, ${ }^{11}$ F. Tessarotto,${ }^{24}$ A. Thiel, ${ }^{3}$ J. Tomsa,${ }^{17}$ F. Tosello, ${ }^{26}$ V. Tskhay, ${ }^{14}$ S. Uhl, ${ }^{15}$ A. Vauth, ${ }^{9}$ J. Veloso, ${ }^{1}$ M. Virius, ${ }^{18}$ M. Vit, ${ }^{25}$ J. Vondra, ${ }^{18}$ S. Wallner, ${ }^{15}$ T. Weisrock, ${ }^{12}$ M. Wilfert, ${ }^{12}$ J. ter Wolbeek, ${ }^{8}$ K. Zaremba, ${ }^{30}$ P. Zavada, ${ }^{7}$ M. Zavertyaev, ${ }^{14}$ E. Zemlyanichkina, ${ }^{7}$ N. Zhuravlev, ${ }^{7}$ and M. Ziembicki ${ }^{30}$

\section{(COMPASS Collaboration)}

\author{
${ }^{1}$ University of Aveiro, Department of Physics, 3810-193 Aveiro, Portugal \\ ${ }^{2}$ Universität Bochum, Institut für Experimentalphysik, 44780 Bochum, Germany \\ ${ }^{3}$ Universität Bonn, Helmholtz-Institut für Strahlen- und Kernphysik, 53115 Bonn, Germany \\ ${ }^{4}$ Universität Bonn, Physikalisches Institut, 53115 Bonn, Germany \\ ${ }^{5}$ Institute of Scientific Instruments, AS CR, 61264 Brno, Czech Republic \\ ${ }^{6}$ Matrivani Institute of Experimental Research and Education, Calcutta 700 030, India \\ ${ }^{7}$ Joint Institute for Nuclear Research, 141980 Dubna, Moscow region, Russia \\ ${ }^{8}$ Universität Freiburg, Physikalisches Institut, 79104 Freiburg, Germany \\ ${ }^{9}$ CERN, 1211 Geneva 23, Switzerland \\ ${ }^{10}$ Technical University in Liberec, 46117 Liberec, Czech Republic \\ ${ }^{11}$ LIP, 1000-149 Lisbon, Portugal \\ ${ }^{12}$ Universität Mainz, Institut für Kernphysik, 55099 Mainz, Germany \\ ${ }^{13}$ University of Miyazaki, Miyazaki 889-2192, Japan \\ ${ }^{14}$ Lebedev Physical Institute, 119991 Moscow, Russia \\ ${ }^{15}$ Technische Universität München, Physik Department, 85748 Garching, Germany \\ ${ }^{16}$ Nagoya University, 464 Nagoya, Japan \\ ${ }^{17}$ Charles University in Prague, Faculty of Mathematics and Physics, 18000 Prague, Czech Republic \\ ${ }^{18}$ Czech Technical University in Prague, 16636 Prague, Czech Republic
}


${ }^{19}$ State Scientific Center Institute for High Energy Physics of National Research Center “Kurchatov Institute,” 142281 Protvino, Russia

${ }^{20}$ IRFU, CEA, Université Paris-Saclay, 91191 Gif-sur-Yvette, France

${ }^{21}$ Academia Sinica, Institute of Physics, Taipei 11529, Taiwan

${ }^{22}$ Tel Aviv University, School of Physics and Astronomy, 69978 Tel Aviv, Israel

${ }^{23}$ University of Trieste, Department of Physics, 34127 Trieste, Italy

${ }^{24}$ Trieste Section of INFN, 34127 Trieste, Italy

${ }^{25}$ University of Turin, Department of Physics, 10125 Turin, Italy

${ }^{26}$ Torino Section of INFN, 10125 Turin, Italy

${ }^{27}$ University of Illinois at Urbana-Champaign, Department of Physics, Urbana, Illinois 61801-3080, USA

${ }^{28}$ National Centre for Nuclear Research, 00-681 Warsaw, Poland

${ }^{29}$ University of Warsaw, Faculty of Physics, 02-093 Warsaw, Poland

${ }^{30}$ Warsaw University of Technology, Institute of Radioelectronics, 00-665 Warsaw, Poland

${ }^{31}$ Yamagata University, Yamagata 992-8510, Japan

(Received 13 April 2017; revised manuscript received 13 June 2017; published 12 September 2017)

The first measurement of transverse-spin-dependent azimuthal asymmetries in the pion-induced DrellYan (DY) process is reported. We use the CERN SPS $190 \mathrm{GeV} / c \pi^{-}$beam and a transversely polarized ammonia target. Three azimuthal asymmetries giving access to different transverse-momentum-dependent (TMD) parton distribution functions (PDFs) are extracted using dimuon events with invariant mass between $4.3 \mathrm{GeV} / c^{2}$ and $8.5 \mathrm{GeV} / c^{2}$. Within the experimental uncertainties, the observed sign of the Sivers asymmetry is found to be consistent with the fundamental prediction of quantum chromodynamics (QCD) that the Sivers TMD PDFs extracted from DY have a sign opposite to the one extracted from semiinclusive deep-inelastic scattering (SIDIS) data. We present two other asymmetries originating from the pion Boer-Mulders TMD PDFs convoluted with either the nucleon transversity or pretzelosity TMD PDFs. A recent COMPASS SIDIS measurement was obtained at a hard scale comparable to that of these DY results. This opens the way for possible tests of fundamental QCD universality predictions.

DOI: 10.1103/PhysRevLett.119.112002

According to quantum chromodynamics (QCD), the theory of strong interactions, the internal structure of hadrons explored in hard (semi-)inclusive scattering is described by parton distribution functions (PDFs). For a polarized nucleon, within the twist-2 approximation there are eight transverse-momentum-dependent (TMD) PDFs describing the distributions of longitudinal and transverse momenta of partons and their correlations with nucleon and quark polarizations. These PDFs can be experimentally accessed in hadron-hadron and lepton-hadron collisions; for recent reviews see, e.g., Refs. [1-3]. In this Letter, we consider the Drell-Yan process, i.e., massive lepton-pair production in hadron-nucleon collisions $(h N \rightarrow \ell \bar{\ell} X)$, hereafter referred to as DY, and semi-inclusive hadron measurements in deep-inelastic lepton-nucleon scattering $\left(\ell N \rightarrow \ell^{\prime} h X\right)$, hereafter referred to as SIDIS. For the DY and SIDIS cross sections, TMD factorization was proven to hold [4], which allows one to express them as convolutions of hard-scale dependent TMD PDFs, perturbatively calculable hard-scattering parton cross sections and (for SIDIS) parton fragmentation functions. The hard-scale $Q$ in DY is

Published by the American Physical Society under the terms of the Creative Commons Attribution 4.0 International license. Further distribution of this work must maintain attribution to the author(s) and the published article's title, journal citation, and DOI. given by the invariant mass of the lepton pair and in SIDIS by the square root of the virtuality of the photon exchanged in the DIS process.

The Sivers function [5] plays an important role among the TMD PDFs. It describes the left-right asymmetry in the distribution of unpolarized partons in the nucleon with respect to the plane spanned by the momentum and spin vectors of the nucleon. One of the recent significant theoretical advances in the TMD framework of QCD is the prediction that the two naively time-reversal odd TMD PDFs, i.e., the quark Sivers functions $f_{1 T}^{\perp}$ and Boer-Mulders functions $h_{1}^{\perp}$, have opposite sign when measured in SIDIS on the one hand and in DY or $W$ - and $Z$-boson production on the other [6-8]. The experimental test of this fundamental prediction, which is a direct consequence of QCD gauge invariance, is a major challenge in hadron physics.

Nonzero quark Sivers TMD PDFs have been extracted from SIDIS single-differential results of HERMES [9], COMPASS [10-13], and JLab [14] using both collinear $[15,16]$ and TMD evolution approaches [17-21]. The first measurement of the Sivers effect in $W$ - and Z-boson production in collisions of transversely polarized protons at relativistic heavy ion collider (RHIC) was reported by the STAR Collaboration [22]; the hard scales of these measurements is $Q \approx 80 \mathrm{GeV} / c$ and $90 \mathrm{GeV} / c$. It is quite different from the one explored in fixed-target experiments where $Q$ ranges approximately between $1 \mathrm{GeV} / c$ and $9 \mathrm{GeV} / c$. Hence it is not excluded that TMD evolution 
effects may be sizable when describing the STAR results using Sivers TMD PDFs extracted from fixed-target SIDIS results.

The COMPASS experiment at CERN $[23,24]$ has the unique capability to explore the transverse-spin structure of the nucleon in a similar kinematic region by two alternative experimental approaches, i.e., SIDIS and DY, using mostly the same setup. This offers the opportunity of minimizing uncertainties of TMD evolution in the comparison of the Sivers TMD PDFs when extracted from these two measurements to test the opposite-sign prediction by QCD.

Recently, COMPASS published the first multidifferential results of the Sivers asymmetry, which were extracted from SIDIS data at four different hard scales [25]. In particular for the range $4 \mathrm{GeV} / c<Q<9 \mathrm{GeV} / c$, the Sivers asymmetry for positive hadrons was found to be above 0 by 3.2 standard deviations of the total experimental accuracy. This hard-scale range is very similar to the one used in this Letter to analyze the DY process.

When the polarizations of the produced leptons are summed over, the general expression for the cross section of pion-nucleon DY lepton-pair production off a transversely polarized nucleon comprises five transverse spindependent asymmetries (TSAs), including the Sivers TSA. Those three TSAs that can be described by contributions from only twist-2 TMD PDFs will be addressed in this Letter. Following the conventions of Refs. [24,26], the corresponding part of the differential cross section can be written as follows:

$$
\begin{aligned}
\frac{d \sigma}{d q^{4} d \Omega} \propto & \hat{\sigma}_{U}\left(1+S_{T}\left\{D_{1} A_{T}^{\sin \varphi_{S}} \sin \varphi_{S}\right.\right. \\
& +D_{2}\left[A_{T}^{\sin \left(2 \varphi_{\mathrm{CS}}-\varphi_{S}\right)} \sin \left(2 \varphi_{\mathrm{CS}}-\varphi_{S}\right)\right. \\
& \left.\left.\left.+A_{T}^{\sin \left(2 \varphi_{\mathrm{CS}}+\varphi_{S}\right)} \sin \left(2 \varphi_{\mathrm{CS}}+\varphi_{S}\right)\right]\right\}\right) .
\end{aligned}
$$

Here $q$ is the four-momentum of the exchanged virtual photon and $\hat{\sigma}_{U}=\left(F_{U}^{1}+F_{U}^{2}\right)\left(1+\lambda \cos ^{2} \theta_{\mathrm{CS}}\right)$, with $F_{U}^{1}, F_{U}^{2}$ being the polarization and azimuth-independent structure functions, and the polar asymmetry $\lambda$ is given as $\lambda=\left(F_{U}^{1}-F_{U}^{2}\right) /\left(F_{U}^{1}+F_{U}^{2}\right)$. At the leading order of perturbative QCD, within the twist-2 approximation, $F_{U}^{2}=0$, and therefore $\lambda=1$. The subscript $(U) T$ denotes transverse polarization (in)dependence. In analogy to SIDIS, the virtual-photon depolarization factors are defined as $D_{1}=\left(1+\cos ^{2} \theta_{\mathrm{CS}}\right) /\left(1+\lambda \cos ^{2} \theta_{\mathrm{CS}}\right)$ and $D_{2}=\sin ^{2} \theta_{\mathrm{CS}} /$ $\left(1+\lambda \cos ^{2} \theta_{\mathrm{CS}}\right)$. The angles $\varphi_{\mathrm{CS}}, \theta_{\mathrm{CS}}$, and $\Omega$, the solid angle of the lepton, are defined in the Collins-Soper frame as defined in Refs. [24,26], and $\varphi_{S}$ is the azimuthal angle of the direction of the nucleon polarization in the target rest frame; see Fig. 1.

The TSAs $A_{T}^{w}$ in Eq. (1) are defined as amplitudes of a given azimuthal modulation $w=w\left(\varphi_{S}, \varphi_{\mathrm{CS}}\right)$, divided by the spin and azimuth-independent part of the DY cross section and the corresponding depolarization factor.

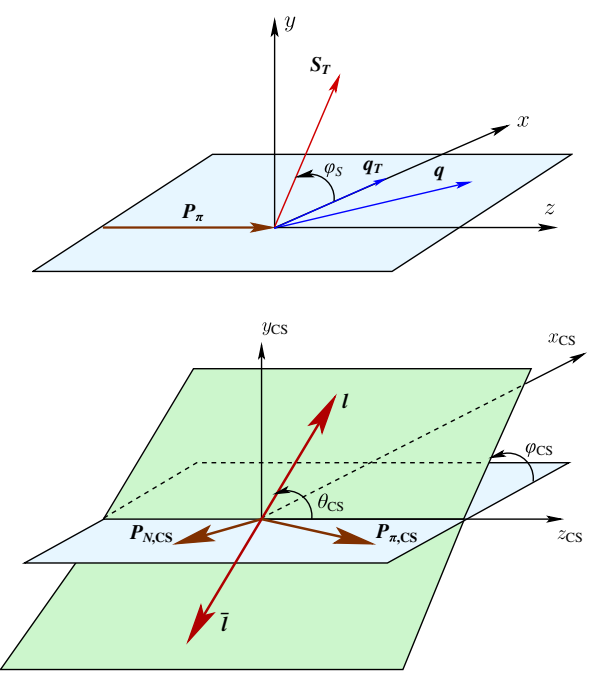

FIG. 1. Top: target rest frame.

In this analysis, the sign convention for TSAs is given by Eq. (1) together with the definitions of azimuthal and polar angles in Fig. 1. Note that the sign of the Sivers TSA is related to that of the Sivers TMD PDF only by the convention that fixes the direction of the $z$-axis, so that the above mentioned sign-change prediction for the Sivers TMD PDFs in our case results in the same sign as measured Sivers TSAs in SIDIS and DY.

In DY lepton-pair production with a transversely polarized nucleon in the initial state, the TSA $A_{T}^{\sin \varphi_{S}}$ is related to the nucleon Sivers TMD PDFs $\left(f_{1 T}^{\perp}\right)$ convoluted with the unpolarized pion TMD PDFs $\left(f_{1, \pi}\right)$. The other two TSAs, $A_{T}^{\sin \left(2 \varphi_{\mathrm{CS}}-\varphi_{S}\right)}$ and $A_{T}^{\sin \left(2 \varphi_{\mathrm{CS}}+\varphi_{S}\right)}$, are related to convolutions of the Boer-Mulders TMD PDFs $\left(h_{1, \pi}^{\perp}\right)$ of the pion with the nucleon TMD PDFs transversity $\left(h_{1}\right)$ and pretzelosity $\left(h_{1 T}^{\perp}\right)$, respectively [26,27]. All three aforementioned nucleon TMD PDFs induce analogous twist-2 TSAs in the general expression for the cross section of unpolarized-hadron production in SIDIS of leptons off transversely polarized nucleons [26-28]. These TSAs were studied by the HERMES and COMPASS experiments [9,11-13,25,29-34]. In contrast to the Sivers function, transversity and pretzelosity are predicted to be genuinely universal; i.e., they do not change sign between SIDIS and DY [4], which is yet another fundamental QCD prediction to be explored.

The analysis presented in this Letter is based on DrellYan data collected by COMPASS in the year 2015 using essentially the same spectrometer as was used during SIDIS data taking in previous years [23]. For this measurement, the $190 \mathrm{GeV} / c \pi^{-}$beam with an average intensity of $0.6 \times 10^{8} \mathrm{~s}^{-1}$ from the CERN SPS was scattered off the COMPASS transversely polarized $\mathrm{NH}_{3}$ target with proton polarization $\left\langle P_{T}\right\rangle \approx 0.73$ and dilution factor $\langle f\rangle \approx 0.18$, where the latter accounts for the fraction of polarizable nucleons in the target and the migration of reconstructed 
events from one target cell to the other. The polarized target, placed in a $0.6 \mathrm{~T}$ dipole magnet, consisted of two longitudinally aligned cylindrical cells of $55 \mathrm{~cm}$ in length and $4 \mathrm{~cm}$ in diameter, separated by a $20 \mathrm{~cm}$ gap. The two cells were polarized vertically in opposite directions, so that data with both spin orientations were recorded simultaneously. In order to compensate for acceptance effects, the polarization was reversed every two weeks. The entire datataking time of 18 weeks was divided into nine periods, each consisting of two consecutive weeks with opposite target polarizations. The proton polarization had a relaxation time of about 1000 hours, which was measured for each target cell in each data-taking period. A $240 \mathrm{~cm}$ long structure made mostly of alumina with a tungsten core, placed downstream of the target, acted as hadron absorber and beam dump. Outgoing charged particles were detected by a system of tracking detectors in the two-stage spectrometer. In each stage, muon identification was accomplished by a system of muon filters.

The trigger required the hit pattern of several hodoscope planes to be consistent with at least two muon candidates originating from the target region. For any pair of candidates, either both have to be detected in the first stage of the spectrometer (25 $\mathrm{mrad}<\theta_{\mu}<160 \mathrm{mrad}$ ) or one has to be detected in the first and the other in the second stage ( $8 \mathrm{mrad}<\theta_{\mu}<45 \mathrm{mrad}$ ).

In the data analysis, the selection of events requires a production vertex located within the polarized-target volume, with one incoming pion beam track and at least two oppositely charged outgoing particles that are consistent with the muon hypothesis; i.e., they crossed at least 30 radiation lengths of material along the spectrometer. The dimuon transverse momentum $q_{T}$ is required to be above $0.4 \mathrm{GeV} / c$ in order to obtain sufficient resolution in angular variables.

The reconstructed mass spectrum of events passing all analysis requirements is shown in Fig. 2 (in black). The combinatorial background originating from the decays of pions and kaons produced in the target is evaluated using like-sign dimuon events from real data and is shown in gray (dotted). Further contributions to the dimuon spectrum are

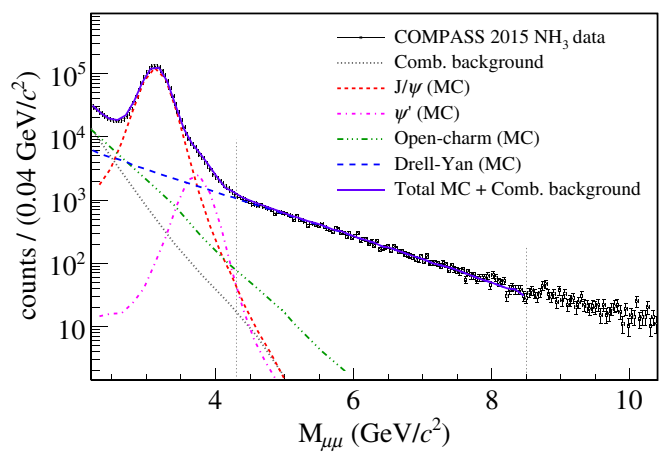

FIG. 2. The dimuon invariant mass distribution. evaluated with a Monte Carlo (MC) simulation, and their relative weights are obtained by a fit to the data. The DrellYan contribution is shown in blue (long dashed). The background contributions originate from charmonia, shown in red (dashed) and magenta (dot-dashed), and semimuonic open-charm decays shown in green (double dot dashed). The sum of all contributions, shown in violet, describes the experimental data well. The $J / \psi$ peak is clearly visible with a shoulder from the $\psi(2 S)$ resonance. For the analysis we use the mass range $4.3 \mathrm{GeV} / c^{2}<M_{\mu \mu}<8.5 \mathrm{GeV} / c^{2}$, where the upper limit avoids the contribution of $\Upsilon$ resonances. In this range, the sum of all background contributions is estimated to be below $4 \%$.

The two-dimensional distribution of the Bjorken scaling variables of pion and nucleon, $x_{\pi}$ and $x_{N}$, for this mass range is presented in Fig. 3. The figure shows that the kinematic phase space explored by the COMPASS spectrometer matches the valence region in $x_{\pi}$ and $x_{N}$. In this region, the DY cross section for a proton target is dominated by the contribution of nucleon $u$-quark and pion $\bar{u}$-quark TMD PDFs.

The distributions of the dimuon Feynman variable $x_{F}$ and the dimuon transverse momentum $q_{T}$ are presented in Fig. 4. The corresponding mean values of the kinematic variables are $\left\langle x_{N}\right\rangle=0.17,\left\langle x_{\pi}\right\rangle=0.50,\left\langle x_{F}\right\rangle=0.33$, $\left\langle q_{T}\right\rangle=1.2 \mathrm{GeV} / c$, and $\left\langle M_{\mu \mu}\right\rangle=5.3 \mathrm{GeV} / c^{2}$.

About $35 \times 10^{3}$ dimuons remain for the analysis. The three TSAs presented in this Letter are extracted period by period from the number of dimuons produced in each cell for the two directions of the target polarization. The doublecell target configuration in conjunction with the periodic polarization reversal allows for the simultaneous measurement of azimuthal asymmetries for both target spin orientations. Using an extended unbinned maximum likelihood estimator, all five TSAs are fitted simultaneously together with their correlation matrices. In this approach, flux and acceptance-dependent systematic uncertainties are minimized [31]. The final asymmetries are obtained by averaging the results of the nine periods. The asymmetries are evaluated in kinematic bins of $x_{N}, x_{\pi}, x_{F}$, or $q_{T}$, while always integrating over all the other variables.

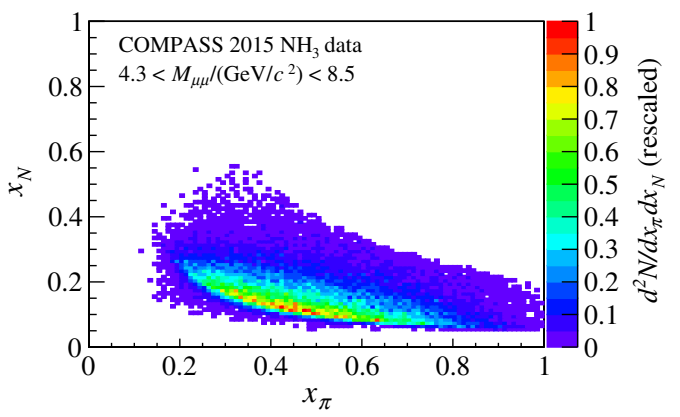

FIG. 3. The two-dimensional $\left(x_{\pi}, x_{N}\right)$ distribution of the selected high mass dimuons. The distribution is normalized to have a maximum value equal to one. 

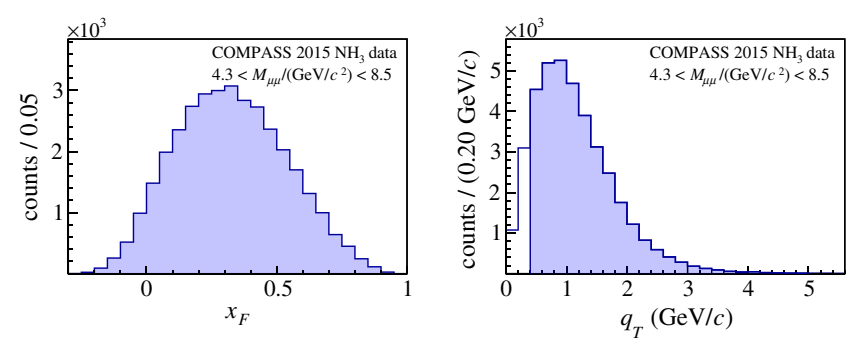

FIG. 4. The $x_{F}$ distribution (left) and $q_{T}$ distribution (right) of the selected high mass dimuons.

The dilution factor $f$ and the depolarization factor $D_{2}$ entering the definition of TSAs are calculated on an eventby-event basis and are used to weight the asymmetries. For the magnitude of the target polarization $P_{T}$, an average value is used for each data-taking period in order to avoid possible systematic bias. In the evaluation of the depolarization factors, the approximation $\lambda=1$ is used. Known deviations from this assumption with $\lambda$ ranging between 0.5 and $1[35,36]$ decrease the normalization factor by at most $5 \%$.

The TSAs resulting from different periods are checked for possible systematic effects. The largest systematic uncertainty is due to possible residual variations of experimental conditions within a given period. They are quantified by evaluating various types of false asymmetries in a similar way as described in Refs. [12,30]. The systematic point-to-point uncertainties are found to be about 0.7 times the statistical uncertainties. The normalization uncertainties originating from the uncertainties on target polarization $(5 \%)$ and dilution factor $(8 \%)$ are not included in the quoted systematic uncertainties.

The TSAs $A_{T}^{\sin \varphi_{S}}, A_{T}^{\sin \left(2 \varphi_{\mathrm{CS}}-\varphi_{S}\right)}$, and $A_{T}^{\sin \left(2 \varphi_{\mathrm{CS}}+\varphi_{S}\right)}$ are shown in Fig. 5 as a function of the variables $x_{N}, x_{\pi}$, $x_{F}$, and $q_{T}$. Because of relatively large statistical uncertainties, no clear trend is observed for any of the TSAs. The full set of numerical values for all TSAs, including correlation coefficients and mean kinematic values from this measurement, is available on HepData [37]. The last column in Fig. 5 shows the results for the three extracted TSAs integrated over the entire kinematic range. The average Sivers asymmetry $A_{T}^{\sin \varphi_{S}}=0.060 \pm 0.057$ (stat) \pm 0.040 (sys) is found to be above 0 at about one standard deviation of the total uncertainty. In Fig. 6, it is compared with recent theoretical predictions from Refs. [19-21] that are based on standard DGLAP and two different TMD evolution approaches. (Note that the kinematic constraints used in Refs. [19-21] differ from one another and also from those used in our analysis.) The positive sign of these theoretical predictions for the DY Sivers asymmetry was obtained by using the sign-change hypothesis for the Sivers TMD PDFs, and the numerical values are based on a fit of SIDIS data for the Sivers TSA $[9,11,12]$. Figure 6 shows that this first measurement of the DY Sivers asymmetry is

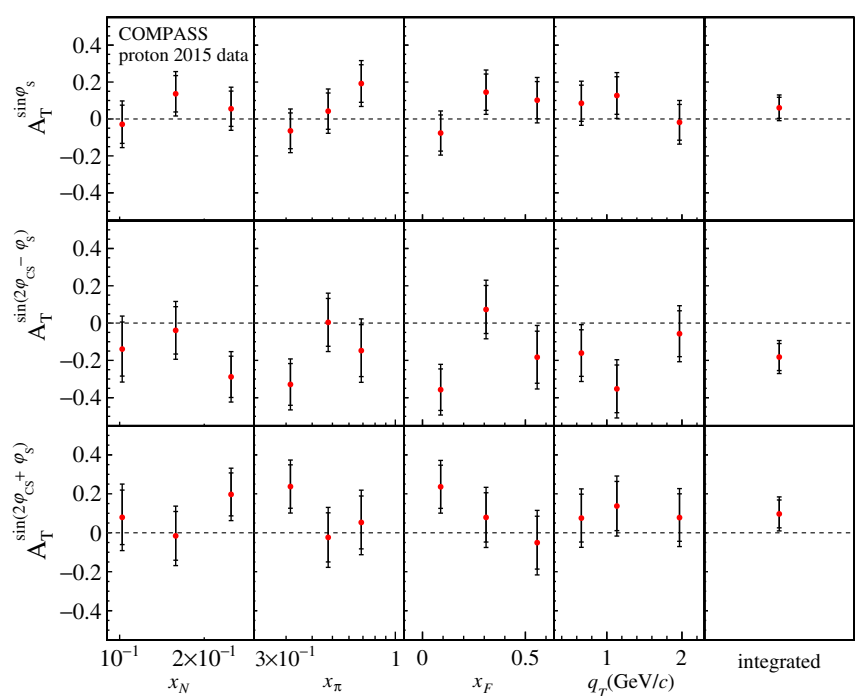

FIG. 5. Extracted Drell-Yan TSAs related to Sivers, transversity, and pretzelosity TMD PDFs (top to bottom). Inner (outer) error bars represent statistical (total experimental) uncertainties. The normalization uncertainties due to target polarization $(5 \%)$ and dilution factor $(8 \%)$ are not included in the error bars.

consistent with the predicted change of sign for the Sivers function.

The average value for the TSA $A_{T}^{\sin \left(2 \varphi_{\mathrm{CS}}-\varphi_{S}\right)}$ is measured to be below 0 with a significance of about two standard deviations. The obtained magnitude of the asymmetry is in agreement with the model calculations of Ref. [38] and can be used to study the universality of the nucleon transversity function. The TSA $A_{T}^{\sin \left(2 \varphi_{\mathrm{CS}}+\varphi_{S}\right)}$, which is related to the nucleon pretzelosity TMD PDFs, is measured to be above 0 with a significance of about one standard deviation. Since both $A_{T}^{\sin \left(2 \varphi_{\mathrm{CS}}-\varphi_{S}\right)}$ and $A_{T}^{\sin \left(2 \varphi_{\mathrm{CS}}+\varphi_{S}\right)}$ are related to the pion Boer-Mulders PDFs, the obtained results may be used to study this function further and to possibly determine its

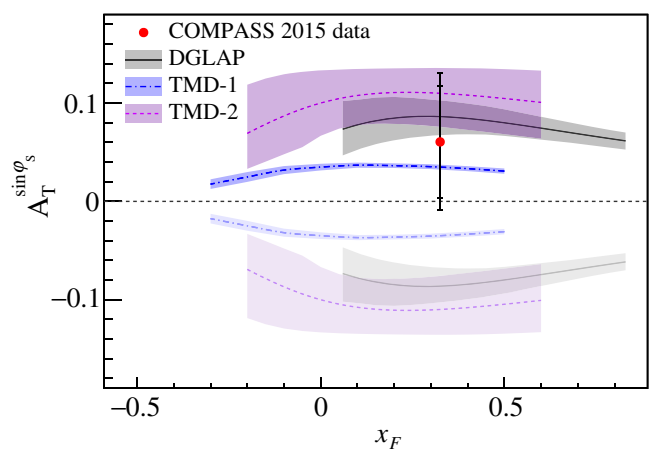

FIG. 6. The measured mean Sivers asymmetry and the theoretical predictions for different $Q^{2}$ evolution schemes from Refs. [19] (DGLAP), [20] (TMD1), and [21] (TMD2). The dark-shaded (light-shaded) predictions are evaluated with (without) the sign-change hypothesis. Uncertainties are as described in Fig. 5. 
sign. They may also be used to test the sign change of the nucleon Boer-Mulders TMD PDFs between SIDIS and DY as predicted by QCD [6-8], when combined with other past and future SIDIS and DY data related to target-spinindependent Boer-Mulders asymmetries [39-41]. In 2018, COMPASS will continue the measurements of the polarized Drell-Yan process for another data-taking year.

We gratefully acknowledge the support of the CERN management and staff and the skill and effort of the technicians of our collaborating institutes. Special thanks go to A. Dudarev, E. Feldbaumer, L. Gatignon, C. Theis, H. Ten Kate, and H. Vincke for invaluable help in the preparation of this experiment. For fruitful discussions and essential input to the preparation of the proposal of this measurement, we thank M. Anselmino, A. Bacchetta, A. Bianconi, S. Melis, M. Radici, O. Teryaev, B. Pasquini, and A. Prokudin. We are grateful to M. E. Boglione, M. Echevarria, and F. Yuan for providing us with numerical values of their model predictions. This work was made possible by the financial support of our funding agencies: MEYS (Czech Republic) Grant No. LG13031; FP7 "HadronPhysics3" Grant No. 283286 (European Union); CEA, P2I and ANR (France); BMBF, DFG Cluster of Excellence "Origin and Structure of the Universe," DFG Research Training Group Programmes 1102 and 2044 (Germany); B. Sen Fund (India); Academy of Sciences and Humanities (Israel); INFN (Italy); MEXT and JSPS, Grants No. 8002006, No. 20540299, and No. 18540281, Daiko and Yamada Foundations (Japan); NCN Grant No. 2015/18/M/ST2/00550 (Poland); FCT COMPETE and QREN, Grants No. CERN/FP 116376/ 2010, No. 123600/2011, and No. CERN/FIS-NUC/0017/ 2015 (Portugal); CERN-RFBR Grant No. 12-02-91500, Presidential Grant No. NSh-999.2014.2 (Russia); MST (Taiwan); and NSF (USA).

\footnotetext{
${ }^{\mathrm{a}}$ Deceased.

${ }^{\mathrm{b}}$ Also at Instituto Superior Técnico, Universidade de Lisboa, Lisbon, Portugal.

${ }^{c}$ Also at Department of Physics, Pusan National University, Busan 609-735, Republic of Korea and at Physics Department, Brookhaven National Laboratory, Upton, NY 11973, USA.

${ }^{\mathrm{d}}$ Also at Abdus Salam ICTP, 34151 Trieste, Italy.

${ }^{\mathrm{e}}$ Retired from Ludwig-Maximilian-Universität, München, Germany.

${ }^{\mathrm{f}}$ Also at Chubu University, Kasugai, Aichi 487-8501, Japan. ${ }^{g}$ Also at Department of Physics, National Central University, 300 Jhongda Road, Jhongli 32001, Taiwan.

${ }^{\mathrm{h}}$ Also at KEK, 1-1 Oho, Tsukuba, Ibaraki 305-0801, Japan. IAlso at Moscow Institute of Physics and Technology, Moscow region, 141700, Russia.

${ }^{\mathrm{j}}$ Also at Department of Physics, National Kaohsiung Normal University, Kaohsiung County 824, Taiwan.

${ }^{\mathrm{k}}$ Also at University of Eastern Piedmont, 15100 Alessandria, Italy.
}

'Present address: RWTH Aachen University, III. Physikalisches Institut, 52056 Aachen, Germany.

${ }^{\mathrm{m}}$ Present address: Uppsala University, Box 516, 75120 Uppsala, Sweden.

[1] M. G. Perdekamp and F. Yuan, Annu. Rev. Nucl. Part. Sci. 65, 429 (2015).

[2] J.-C. Peng and J.-W. Qiu, Prog. Part. Nucl. Phys. 76, 43 (2014).

[3] C. A. Aidala, S. D. Bass, D. Hasch, and G. K. Mallot, Rev. Mod. Phys. 85, 655 (2013).

[4] J. Collins, Foundations of Perturbative QCD (Cambridge University Press, Cambridge, England, 2013).

[5] D. W. Sivers, Phys. Rev. D 41, 83 (1990).

[6] J. C. Collins, Phys. Lett. B 536, 43 (2002).

[7] S. J. Brodsky, D. S. Hwang, and I. Schmidt, Phys. Lett. B 530, 99 (2002).

[8] S. J. Brodsky, D. S. Hwang, and I. Schmidt, Nucl. Phys. B642, 344 (2002).

[9] A. Airapetian et al. (HERMES Collaboration), Phys. Rev. Lett. 103, 152002 (2009).

[10] E. S. Ageev et al. (COMPASS Collaboration), Nucl. Phys. B765, 31 (2007).

[11] M. Alekseev et al. (COMPASS Collaboration), Phys. Lett. B 673, 127 (2009).

[12] C. Adolph et al. (COMPASS Collaboration), Phys. Lett. B 717, 383 (2012).

[13] C. Adolph et al. (COMPASS Collaboration), Phys. Lett. B 744, 250 (2015).

[14] X. Qian et al. (Jefferson Lab Hall A Collaboration), Phys. Rev. Lett. 107, 072003 (2011).

[15] M. Anselmino, M. Boglione, U. D'Alesio, A. Kotzinian, F. Murgia, and A. Prokudin, Phys. Rev. D 72, 094007 (2005)

[16] M. Anselmino, M. Boglione, U. D’Alesio, A. Kotzinian, S. Melis, F. Murgia, A. Prokudin, and C. Turk, Eur. Phys. J. A 39, 89 (2009).

[17] S. M. Aybat, A. Prokudin, and T. C. Rogers, Phys. Rev. Lett. 108, 242003 (2012).

[18] M. Anselmino, M. Boglione, and S. Melis, Phys. Rev. D 86, 014028 (2012).

[19] M. Anselmino, M. Boglione, U. D'Alesio, F. Murgia, and A. Prokudin, J. High Energy Phys. 04 (2017) 046.

[20] M. G. Echevarria, A. Idilbi, Z.-B. Kang, and I. Vitev, Phys. Rev. D 89, 074013 (2014).

[21] P. Sun and F. Yuan, Phys. Rev. D 88, 114012 (2013).

[22] L. Adamczyk et al. (STAR Collaboration), Phys. Rev. Lett. 116, 132301 (2016).

[23] P. Abbon et al. (COMPASS Collaboration), Nucl. Instrum. Methods Phys. Res., Sect. A 577, 455 (2007).

[24] F. Gautheron et al. (COMPASS Collaboration), Report No. SPSC-P-340, CERN-SPSC-2010-014, 2010.

[25] C. Adolph et al. (COMPASS Collaboration), Phys. Lett. B 770, 138 (2017).

[26] S. Arnold, A. Metz, and M. Schlegel, Phys. Rev. D 79, 034005 (2009).

[27] A. Bacchetta, M. Diehl, K. Goeke, A. Metz, P. J. Mulders, and M. Schlegel, J. High Energy Phys. 02 (2007) 093.

[28] A. Kotzinian, Nucl. Phys. B441, 234 (1995).

[29] C. Adolph et al. (COMPASS Collaboration), Phys. Lett. B 736, 124 (2014). 
[30] C. Adolph et al. (COMPASS Collaboration), Phys. Lett. B 717, 376 (2012).

[31] C. Adolph et al. (COMPASS Collaboration), Phys. Lett. B 713, 10 (2012).

[32] B. Parsamyan et al. (COMPASS Collaboration), Phys. Part. Nucl. 45, 158 (2014).

[33] L. L. Pappalardo et al. (HERMES Collaboration), in Exclusive Reactions at High Momentum Transfer. Proceedings, 4th Workshop, Newport News, VA, 2010 (World Scientific Publishing Co Pte Ltd, Singapore, Singapore, 2011), pp. 312-320.

[34] F. Bradamante et al. (COMPASS Collaboration), arXiv:1702.00621.

[35] M. Guanziroli et al. (NA10 Collaboration), Z. Phys. C 37, 545 (1988).
[36] J. S. Conway, C. E. Adolphsen, J. P. Alexander, K. J. Anderson, J. G. Heinrich, J. E. Pilcher, A. Possoz, E. I. Roseberg, C. Biino, J. F. Greenhalgh, W. C. Louis, K. T. McDonald, S. Palestini, F. C. Shoemaker, and A. J. S. Smith, Phys. Rev. D 39, 92 (1989).

[37] HepData (The Durham HepData Project) (2016).

[38] A. N. Sissakian, O. Yu. Shevchenko, A. P. Nagaitsev, and O. N. Ivanov, Phys. Part. Nucl. 41, 64 (2010).

[39] S. Falciano et al. (NA10 Collaboration), Z. Phys. C 31, 513 (1986).

[40] A. Airapetian et al. (HERMES Collaboration), Phys. Rev. D 87, 012010 (2013).

[41] C. Adolph et al. (COMPASS Collaboration), Nucl. Phys. B886, 1046 (2014). 